\title{
PERTURBED ALGORITHM AND STABILITY FOR STRONGLY NONLINEAR QUASI-VARIATIONAL INCLUSION INVOLVING $H$-ACCRETIVE OPERATORS
}

\author{
MAO-MING JIN
}

\begin{abstract}
In this paper, a new class of strongly nonlinear quasi-variational inclusions involving $H$-accretive operator in Banach spaces is studied, which includes many variational inequality(inclusion) and complementarity problems as special cases. By using the resolvent operator technique for $H$-accretive operator due to Fang and Huang, an existence and uniqueness theorem of solution for strongly nonlinear quasi-variational inclusion is proved. A new perturbed algorithm for finding approximate solution of the strongly nonlinear quasi-variational inclusion is suggested and discussed, the convergence and stability of the iterative sequence generated by new perturbed algorithm is also given. The results presented in this paper improve and generalize some recent results in this field.
\end{abstract}

Mathematics subject classification (2000): 49J40, 47H17, 47H10.

Key words and phrases: $H$-accretive operator, strongly nonlinear quasi-variational inclusion, resolvent operator technique, perturbed algorithm, stability.

\section{REFERENCES}

[1] S. AdLY, Perturbed algorithm and sensitivity analysis for a general class of variational inclusions, J. Math. Anal. Appl., 201, (1996), 609-630.

[2] R. P. AgARWAL, Y. J. CHO AND N. J. HUANG, Sensitivity analysis for strongly nonlinear quasi-variational inclusions, Appl. Math. Lett. 13, (2000), 19-24.

[3] R. P. Agarwal, N. J. HuAng AND Y. J. CHO, Generalized nonlinear mixed implicit quasi-variational inclusions with set-valued mappings, J. Inequal. Appl., 7, (6) (2002), 807-828.

[4] R. AHMAD, Q. H. ANSARI, An iterative algorithm for generalized nonlinear variational inclusions, Appl. Math. lett. , 13, (5) (2002), 23-26.

[5] S. S. ChANG, Y. J. CHO AND H. Y. ZHOU, Iterative Methods for Nonlinear Operator Equations in Banach Spaces, Nova Sci. Publ., New York, (2002).

[6] J. Y. CHEN, N. C. WONG AND J. C. YAO, Algorithm for generalized co-complementarity problems in Banach spaces, Computers Math. Applic. , 43, (1/2) (2002), 49-54.

[7] X. P. DING, C. L. LUO, Perturbed proximal point algorithms for generalized quasi-variational-like inclusions, J. Comput. Appl. Math., 210, (2000), 153-165.

[8] Y. P. FANG, N. J. HUANG, $H$-Accretive operator and resolvent operator technique for solving variational inclusions in Banach spaces, Appl. Math. Lett., 17, (2004), 647-653.

[9] Y. P. FANG, N. J. HUANG, $H$-monotone operators and system of variational inclusions, Communications on Applied Nonlinear Analysis, 11, (1) (2004), 93-101.

[10] F. Giannessi, A. MaugerI, Variational Inequalities and Network Equilibrium Problems, New York (1995).

[11] A. HaSsounI, A. MoudAFI, A perturbed algorithms for variational inequalities, J. Math. Anal. Appl., 185, (1994), 706-712.

[12] N. J. HUANG, Generalized nonlinear variational inclusions with noncompact valued mapping, Appl. Math. Lett., 9, (3) (1996), 25-29. 
[13] N. J. HuANG, Nonlinear Implicit quasi-variational inclusions involving generalized $m$-accretive mappings, Arch. Inequal. Appl., 2, (2004), 403-416.

[14] N. J. HUANG, Mann and Ishikawa type perturbed iterative algorithms for generalized nonlinear implicit quasi-variational inclusions, Computers Math. Applic., 35, (10) (1998), 1-7.

[15] N. J. HUANG, A new completely general class of variational inclusions with noncompact valued mappings, Computers Math. Applic., 35, (10) (1998), 9-14.

[16] N. J. HUANG, Y. P. FANG, A new class of general variational inclusions involving maximal $\eta$-monotone mappings, Publ. Math. Debrecen, 62, (2003), 83-98.

[17] C. R. JOU, J. C. YAO, Algorithm for generalized multivalued variational inequalities in Hilbert spaces, Computers Math. Applic., 25, (9) (1993), 7-16.

[18] J. S. Jung, C. H. MoRALES, The Mann process for perturbed $m$-accretive operator in Banach spaces, Nonlinear Anal. T. M. A., 46, (2) (2001), 231-243.

[19] M. M. Jin, Sensitivity analysis for strongly nonlinear quasi-variational inclusions involving generalized $m$-accretive mappings, Nonlinear Anal. Forum, 8, (1) (2003), 93-99.

[20] M. M. JIN, Q. K. LIU, Nonlinear quasi-variational inclusions involving generalized $m$-accretive mappings, Nonlinear Funct. Anal. Appl., 9, (3) (2004), 485-494.

[21] M. M. JIN, Generalized nonlinear implicit quasi-variational inclusions with relaxed monotone mappings, Adv. Nonlinear Var. Inequal., 7, (2) (2004), 173-181.

[22] K. R. KAZMI, Mann and Ishikawa type perturbed iterative algorithms for generalized quasivariational inclusions, J. Math. Anal. Appl., 209, (1997), 572-584.

[23] H. Y. LAN, J. K. Kim AND N. J. HuANG, On the generalized nonlinear quasi-variational inclusions involving non-monotone set-valued mappings, Nonlinear Funct. Anal. Appl., 9, (3) (2004), 451-465.

[24] C. H. LEE, Q. H. ANSARI AND J. C. YAO, A perturbed algorithm for strongly nonlinear variational-like inclusions, Bull. Austral. Math. Soc., 62, (2000), 417-426.

[25] S. H. ShIM, S. M. KANG, N. J. HuAng AND J. C. YAO, Perturbed iterative algorithms with errors for completely generalized strongly nonlinear implicit variational-like inclusions, J. Inequal. Appl., 5, (4) (2000), 381-395.

[26] R. U. VERMA, Generalized variational inequalities involving multivalued relaxed monotone operators, Appl. Math. Lett. , 10, (4) (1997), 107-109.

[27] R. U. VERMA, A -monotonicity and applications to nonlinear variational inclusions, Journal of Applied Mathematics and Stochastic Analysis, 17, (2) (2004), 193-195.

[28] R. U. VERMA, Approximation-solvability of a class of A-monotone variational inclusion problems, Journal KSIAM, 8, (1) (2004), 55-66.

[29] X. L. WenG, Fixed point iteration for local strictly pseudo-contractive mapping, Proc. Amer. Math. Soc, 113, (1991), 727-732.

[30] G. X. Z. YUAN, KKM Theory and Applications in Nonlinear Analysis, Marcel Dekker, New York, (1999).

[31] H. K. XU, Inequalities in Banach spaces with applications, Nonlinear Anal., 16, (2) (1991), 1127-1138.

[32] W. Y. YAN, Y. P. FANG AND N. J. HUANG, A new system of set-valued variational inclusions with H-monotone operators, Mathematical Inequalities \& Applications, 8, (3) (2005), 537-546. 